\title{
Ulipristal Acetate Versus Leuprolide Acetate in Medical Management of Uterine Fibroids
}

\author{
Veladanda Kavitha ${ }^{1}$, Rachamalla Madhuri ${ }^{2}$ \\ ${ }^{1,2}$ Department of Obstetrics and Gynaecology, Chalmeda Anand Rao \\ Institute of Medical Sciences, Bommakal, Karimnagar, Telangana, India.
}

\section{ABSTRACT}

\section{BACKGROUND}

We wanted to compare the effectiveness of the treatment and the adverse effects of ulipristal acetate and leuprolide acetate in the medical management of symptomatic uterine fibroids.

\section{METHODS}

This is a randomised controlled study conducted in the the Department of Obstetrics and Gynaecology in Chalmeda Anand Rao Institute of Medical Sciences from January 2019 to January 2020. 60 patients with symptomatic fibroids and excessive uterine bleeding were randomly divided. They were given daily therapy of ulipristal acetate $10 \mathrm{mg}$ orally for 3 months or monthly injection leuprolide acetate $3.75 \mathrm{mg}$ intramuscularly for 3 months.

\section{RESULTS}

Controlled uterine bleeding was observed in $98 \%$ of patients who received oral therapy of ulipristal acetate of $10 \mathrm{mg}$, and $89 \%$ of patients who received injections of leuprolide acetate, for differences in comparison with leuprolide acetate of $8.8 \%$ points (95\% CI, 0.4 to 18.3) for ulipristal acetate of $10 \mathrm{mg}$. Median time of amenorrhea for those taking ulipristal acetate of $10 \mathrm{mg}$ was 5 days, and 21 days for leuprolide acetate. $10 \%$ of patients receiving ulipristal acetate reported moderateto-severe hot flashes and $40 \%$ of patients receiving leuprolide acetate reported moderate to severe hot flashes $(\mathrm{P}<0.0010$ for each dose of leuprolide acetate vs. ulipristal acetate).

\section{CONCLUSIONS}

Daily therapy of 10-mg ulipristal acetate was considered non inferior when compared to monthly injections of leuprolide acetate in control of uterine bleeding, moreover ulipristal acetate therapy was also significantly less likely to cause hot flashes.

\section{KEY WORDS}

Ulipristal Acetate; Leuprolide acetate; Abnormal Uterine Bleeding; Leiomyoma
Corresponding Author:

Dr. Rachamalla Madhuri,

D/o Rachamalla Vinod Kumar,

H. No: 3-10-5, Veerabhadra House,

Baharpet, Hyderabad Chowrasta,

Bhongir - 508116, Telangana, India.

E-mail:

rachamalla.madhuri05@gmail.com

DOI: $10.14260 / j e m d s / 2021 / 749$

How to Cite This Article:

Kavitha V, Madhuri R. Ulipristal acetate versus leuprolide acetate in medical management of uterine fibroids. J Evolution Med Dent Sci 2021;10(43):37013706, DOI: 10.14260/jemds/2021/749

Submission 16-06-2021,

Peer Review 12-08-2021,

Acceptance 18-08-2021,

Published 25-10-2021.

Copyright (c) 2021 Veladanda Kavitha et al. This is an open access article distributed under Creative Commons Attribution License [Attribution 4.0 International (CC BY 4.0)] 


\section{BACKGROUND}

The most common benign tumours of the uterus in reproductive age women are uterine fibroids or leiomyomas. Bleeding heavily can cause anaemia in fibroids, pelvic pressure can cause pain, pain during menses, infertility thereby reducing the quality of life of the patient.1,2

Present management includes mainly surgical or radiological intervention and medical management is limited.3,4 Oral progestin use has not been investigated extensively, but some small studies reported few possibilities of promotion of myoma growth and breakthrough bleeding. The use of intrauterine devices that release progestin may reduce the symptoms. Menorrhagia can be controlled in some patients by the use of IUD's that release progestin but submucosal myomas which are distorted are not excluded in their trial. ${ }^{5}$ In most cases medical therapy generally effective are GnRH agonists.

Before myoma surgery, vaginal bleeding in $85 \%$ of patients with anaemia had been stopped by using $\mathrm{GnRH}$ agonist leuprolide acetate (in a 3.75-mg depot formulation) in a controlled trial. ${ }^{6}$

Hot flashes were reported in $67 \%$ of patients in that trial, as leuprolide acetate suppresses estradiol. Fibroids can be treated using mifepristone and selective progesterone receptor modulators (SPRMs) as suggested by small pilot studies and other uncontrolled trials. ${ }^{7}$ In-vitro and in vivo ulipristal acetate has a potent and selective modulator of progesterone-receptor activity. ${ }^{8}$ Ulipristal acetate has no effect on normal myometrial cells but has effect of antiproliferative, antifibrotic, and proapoptotic action on leiomyoma cells as shown in studies of cultured leiomyoma cells. Induction of amenorrhea may be caused by antiproliferative effect in addition to pharmacodynamic effect on the endometrium of SPRMs. ${ }^{9}$

In women with symptomatic fibroids, fibroid and uterine sizes are reduced by ulipristal acetate as found in small placebo-controlled studies. ${ }^{10}$ Efficacy of reduction of symptoms by ulipristal acetate was assessed in this study.

Before planning surgery for symptomatic fibroids, the side-effect profiles of the two drugs, daily oral ulipristal acetate $(10 \mathrm{mg})$ and monthly intramuscular injection of leuprolide acetate $(3.75 \mathrm{mg})$ in controlling bleeding were compared.

In $90 \%$ of patients receiving $5 \mathrm{mg}$ of ulipristal acetate and in $89 \%$ of those receiving leuprolide acetate and in $98 \%$ of those receiving $10 \mathrm{mg}$ of ulipristal acetate, uterine bleeding was controlled, for differences (as compared with leuprolide acetate) of 1.2 percentage points (95\% confidence interval [CI], -9.3 to 11.8 ) for $5 \mathrm{mg}$ of ulipristal acetate and 8.8 percentage points (95\% CI, 0.4 to 18.3 ) for $10 \mathrm{mg}$ of ulipristal acetate $(2,7)$. For patients who received $5 \mathrm{mg}$ of ulipristal acetate, median time to amenorrhea was 7 days, for those receiving $10 \mathrm{mg}$ of ulipristal acetate was 5 days and for those who received leuprolide acetate was 21 days. In $11 \%$ of patients who received $5 \mathrm{mg}$ of ulipristal acetate, and for 10 $\%$ of those who received $10 \mathrm{mg}$ of ulipristal acetate, and for $40 \%$ of those who received leuprolide acetate moderate-tosevere hot flashes were reported.

\section{METHODS}

A blinded randomized controlled, phase 3 trial was conducted to assess the efficacy and safety of ulipristal acetate, in comparison with leuprolide acetate, in the preoperative treatment of symptomatic fibroids, conducted in the Department of Obstetrics and Gynaecology in Chalmeda Anand Rao Institute of Medical Sciences from January 2019 to January 2020.

Ethical clearance certificate registration numberCAIMS/01/IEC/2021/009

\section{Study Population}

Premenopausal women between the ages of 18 and 50 years with a BMI (the weight in kilograms divided by the square of the height in meters) lying between 18 and 40, having a history of heavy uterine bleeding caused by fibroids, having at least one myoma with measurement of $3 \mathrm{~cm}$ or more in diameter (but no myoma measuring $>10 \mathrm{~cm}$ ), and a uterine size equal to or less than 16 weeks of gestation and all patients who were eligible for surgery were included in this study. Written informed consent was taken from all patients.

\section{Assessment of Uterine Bleeding}

Using the pictorial blood-loss assessment chart (PBAC) we assessed uterine bleeding, which objectively estimates menstrual blood loss. The PBAC scale ranges from 0 to $>500$ (with no defined upper limit), with a higher score indicating heavy bleeding. During the screening, patients were provided with standardized sanitary materials and told to record the number of tampons or pads used and the amount of soiling with blood. Patients were asked to complete the PBAC daily throughout the treatment period to week 13 and for 28 days preceding the no-treatment follow-up visits at weeks 26 and 38. For 4 weeks the PBAC score was calculated from the sum of daily PBAC results for 28 days. PBAC score of more than 100 (during the first 8 days of menstruation), which corresponds to blood loss of more than $80 \mathrm{ml}$ is known as menorrhagia. The eligible criterion was a PBAC score of more than 100 that was taken in this study.

\section{Randomization and Treatment}

Patients were randomly assigned in a 1:1 ratio to receive either a daily oral placebo along with an intramuscular injection of $3.75 \mathrm{mg}$ of leuprolide acetate once monthly or oral ulipristal acetate $10 \mathrm{mg}$ daily along with an IM saline injection once monthly. We randomly assigned 60 patients by stratified random sampling. In excessive uterine bleeding and symptomatic fibroids, they received 3 months of daily therapy with oral ulipristal acetate $10 \mathrm{mg}$ or once-monthly intra- muscular injections of leuprolide acetate (at a dose of $3.75 \mathrm{mg}$ ). Treatment was started with 4 days of ongoing menstrual period and was continued until week 13, after which patients could have surgery. Follow-up visits were scheduled, without further treatment, for weeks 17,26 , and 38. Iron supplementation was left to the discretion of the treating physician. 


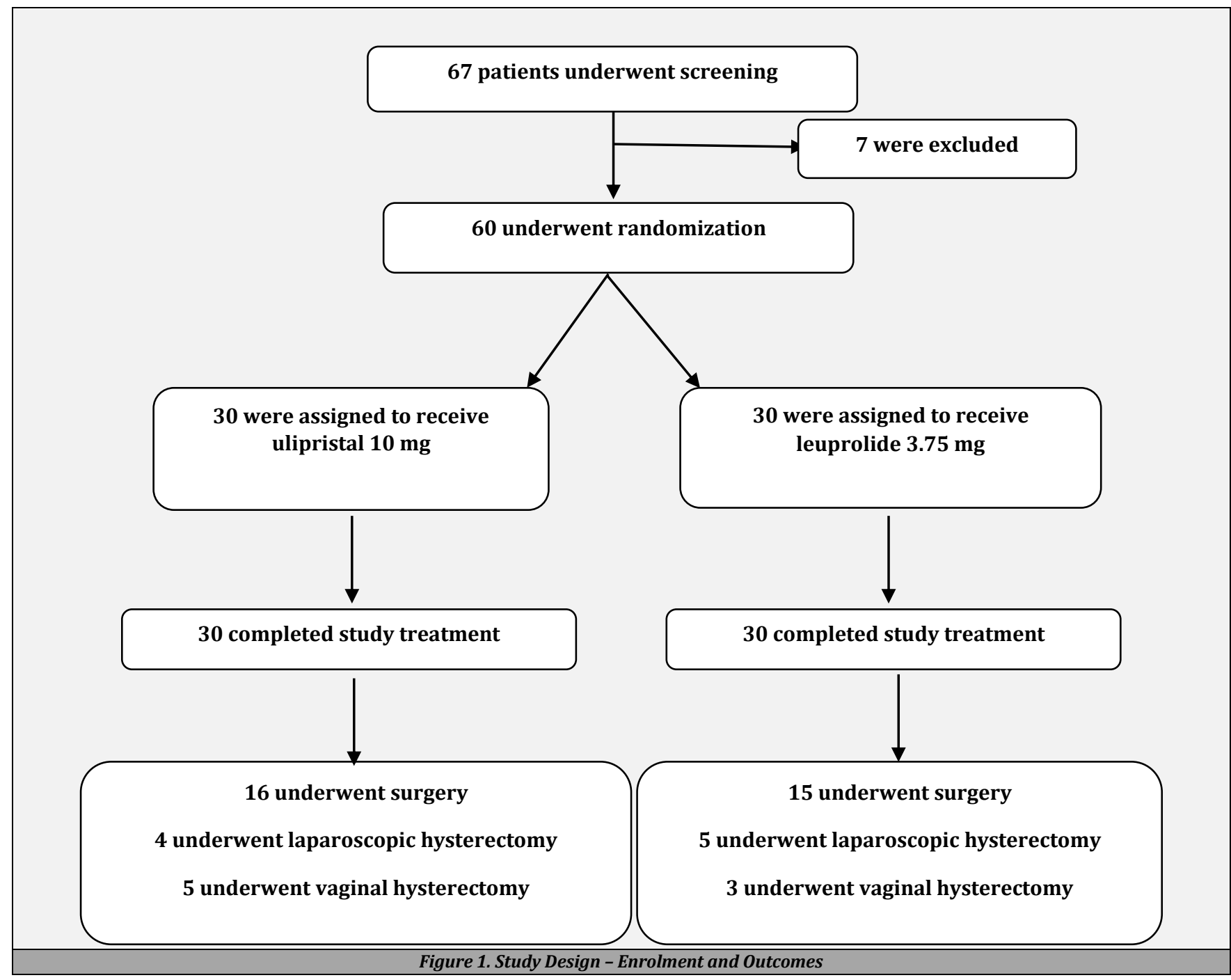

\section{Statistical Analysis}

The sample was taken based on the need to show the noninferiority of ulipristal acetate compared to leuprolide acetate having the power of $90 \%$, using a pre-specified non inferiority with margin of $-20 \%$. And then we summarised quantitative outcomes with mean and standard deviation. The categorical outcomes were summarised in percentages. Numerical variables and categorical variables were compared with the independent sample t-test and chi-square tests respectively.
Two-tailed P-value of $<0.05$ was considered significant. For data analysis, standard statistical software like Microsoft Excel and SPSS version 21.0 was used.

\section{RESULTS}

Demographic and baseline characteristics were balanced among the two study groups.

\begin{tabular}{|c|c|c|c|}
\hline & Characteristics & Ulipristal Acetate & Leuprolide Acetate \\
\hline \multirow{3}{*}{ Safety population } & Patients-number & 30 & 30 \\
\hline & Age-years & $40.75 \pm 6.31$ & $40.34 \pm 6.22$ \\
\hline & Body-mass index & $26.2 \pm 4.7$ & $24.9 \pm 4.1$ \\
\hline \multirow{3}{*}{ Serum estradiol } & Median & 37.0 & 39.0 \\
\hline & Interquartile range & $28.0-59.0$ & $29.0-57.0$ \\
\hline & Endometrial thickness-mm & $8.9 \pm 4.3$ & $9.0 \pm 3.9$ \\
\hline Per-protocol population & Patients-number & 30 & 30 \\
\hline \multirow{2}{*}{ Score on pictorial blood-loss assessment chart } & Median & 271 & 297 \\
\hline & Interquartile range & $183-3922$ & $189-4433$ \\
\hline \multirow{2}{*}{ A total volume of three largest myomas- cucm } & Median & 47.66 & 59.2 \\
\hline & Interquartile range & 24.1-110.6 & $27.8-156.3$ \\
\hline \multirow{3}{*}{ Uterine volume- cucm } & Median & 197.8 & 199.9 \\
\hline & Interquartile range & $120.9-297.7$ & $138.2-271.9$ \\
\hline & Haemoglobin-g/dL & $12.4 \pm 1.6$ & $12.1 \pm 1.8$ \\
\hline \multirow{2}{*}{ Pain assessment - median score(IQR) } & Short- form McGill pain questionnaire & $7.0(4.0-16.0)$ & $7.0(3.0-17.5)$ \\
\hline & Visual- analogue scale & $46.5(20.0-66.5)$ & $46.0(21.0-62.0)$ \\
\hline \multirow{2}{*}{$\begin{array}{c}\text { Uterine fibroid symptom and quality of life } \\
\text { questionnaire }\end{array}$} & severity of a symptom & $48.9 \pm 22.1$ & $52.5 \pm 21.7$ \\
\hline & Health-related quality of life total score & $56.5 \pm 21.4$ & $50.1 \pm 24.9$ \\
\hline
\end{tabular}




\begin{tabular}{|c|c|c|c|c|}
\hline & Variable & Ulipristal Acetate & Leuprolide Acetate & $\begin{array}{l}\text { Difference (Ulipristal Acetate } \\
\text { vs Leuprolide Acetate) }\end{array}$ \\
\hline Per-protocol population & No. of patients & 30 & 30 & \\
\hline \multirow{4}{*}{$\begin{array}{l}\text { Score on pictorial blood- loss } \\
\text { assessment }\end{array}$} & $<75-$ no. / total no. (\%) & $29 / 30(98)$ & $27 / 30(89)$ & $8.8(0.4$ to 18.3$)$ \\
\hline & Median(IQR) & $0(0-0)$ & $0(0-1)$ & \\
\hline & Change from baseline- median (IQR) & $-268(-387$ to -179$)$ & $-274(-430$ to -161$)$ & $3(-45$ to 55$)$ \\
\hline & $\leq 2$, indicating amenorrhea- no. / total no. (\%) & $27 / 30(89)$ & $24 / 30(80)$ & $9.0(-2.8$ to 21.0$)$ \\
\hline \multirow{2}{*}{$\begin{array}{c}\text { Total volume of three largest } \\
\text { myomas }\end{array}$} & $\%$ change from baseline- median (IQR) & $-42(-69$ to -14$)$ & $-53(-69$ to -36$)$ & \\
\hline & Ratio to screening volume- geometric mean & 0.61 & 0.54 & $1.12(0.91$ to 1.38$)$ \\
\hline \multirow{2}{*}{ Uterine volume } & Percentage change from baseline - median (IQR) & $-22(-45$ to 0$)$ & $-47(-57$ to -35$)$ & \\
\hline & Ratio to screening volume - geometric mean & 0.80 & 0.57 & $1.41(1.19$ to 1.66$)$ \\
\hline \multirow{2}{*}{$\begin{array}{l}\text { Short- form McGill pain } \\
\text { questionnaire score }\end{array}$} & Median(IQR) & $1.0(0.0$ to 3.0$)$ & $0.0(0.0$ to 4.0$)$ & \\
\hline & Change from baseline- median (IQR) & $-6.0(-14.0$ to -1.0$)$ & $-5.5(-14.5$ ro -2.0$)$ & $0.0(-2.0$ to 2.8$)$ \\
\hline \multirow{3}{*}{$\begin{array}{l}\text { Uterine fibroid symptom and quality } \\
\text { of life questionnaire }\end{array}$} & Health-related quality of life score & $81.2 \pm 22.1$ & $73.2 \pm 23.0$ & \\
\hline & Change from baseline & $24.8 \pm 24.1$ & $23.2 \pm 28.2$ & $5.6(-3.9$ to 15.1$)$ \\
\hline & Haemoglobin-g/dL & $12.9 \pm 1.2$ & $12.7 \pm 1.6$ & $0.03(-0.3$ to 0.3$)$ \\
\hline Safety population & No. of patients & 30 & 30 & \\
\hline \multirow{5}{*}{ Serum estradiol-pg/mL } & Median & 60.5 & 25.0 & \\
\hline & Interquartile range & 35.0 to 121.0 & 10.0 to 36.0 & \\
\hline & Geometric mean & 69.7 & 24.0 & $2.9(2.3$ to 3.7$)$ \\
\hline & Moderate-to-severe hot flashes- no. (\%) & 3 & 4 & $-29.9(-42.0$ to -16.6$)$ \\
\hline & Endometrial thickness in $\mathrm{mm}$ & $10.7 \pm 5.9$ & $5.1 \pm 3.5$ & $5.6(4.0-7.3)$ \\
\hline
\end{tabular}

\section{Efficacy}

\section{Primary End Point}

In the per-protocol population, proportion of patients having controlled bleeding at week no.13 (PBAC score, $<75$ for the preceding 4 weeks) was $98 \%$ in the group which received 10 mg of ulipristal acetate, and $89 \%$ in the group which received leuprolide acetate. Ulipristal acetate and leuprolide acetate were different by $1.2 \%$ points ( $95 \% \mathrm{CI}, 0.4$ to 18.3 ) for the $10-\mathrm{mg}$ group, indicating noninferiority for both doses of ulipristal acetate in controlling bleeding since the lower margin of the confidence interval for each comparison was more than the pre-specified non-inferiority margin of $-20 \%$. These results were similar to those in the modified intention to treat analysis. In a subsequent superiority analysis, the ulipristal group was superior to the leuprolide group with a 10-mg dose $(\mathrm{P}=0.03)$.

\section{Secondary End Points}

Volume of the 3 largest fibroids was reduced by all the treatments, with median reduction at week 13 of 42 percent in the group which received $10 \mathrm{mg}$ of ulipristal acetate, and 53 percent in the group which received leuprolide acetate. Leuprolide acetate showed more reduction in uterine volume (47\%) than ulipristal group (20 to $22 \%$ ).

Median PBAC scores at the $13^{\text {th }}$ week were 0 for all treatment groups. Significant control of excessive bleeding was achieved much more rapidly in patients who received 10 mg of ulipristal acetate when compared to those who received leuprolide acetate $(\mathrm{P}<0.001$ for both comparisons). Besides the control of excessive bleeding, amenorrhea was induced more rapidly in patients receiving $10 \mathrm{mg}$ of ulipristal acetate than in those who received leuprolide acetate $(\mathrm{P}<$ 0.001). All study groups showed similar improvements in pain, quality of life, and $\mathrm{Hb}$ levels.

At the end of treatment, approximately half of the patients underwent surgery. Another half of the patients who did not undergo surgery in each group showed improvements in bleeding, pain, and quality of life during follow-up without treatment. For patients who did not undergo hysterectomy or myomectomy, ulipristal acetate showed a more sustained effect on the reducing volume of myoma during the following 6 months without treatment than with leuprolide acetate. Menstruation returned on an average of 31 to 34 days after the end of treatment in the ulipristal group and after 43 days in the leuprolide group.

\begin{tabular}{|c|c|c|c|}
\hline & Adverse Events & $\begin{array}{c}\text { Ulipristal } \\
\text { Acetate }(\mathrm{N}= \\
30)\end{array}$ & $\begin{array}{c}\text { Leuprolide } \\
\text { Acetate }(\mathrm{N}= \\
30)\end{array}$ \\
\hline \multirow{9}{*}{$\begin{array}{l}\text { Serious } \\
\text { adverse } \\
\text { events }\end{array}$} & At least one event & $2(6 \%)$ & $2(6 \%)$ \\
\hline & Any event during treatment & $1(3 \%)$ & $1(3 \%)$ \\
\hline & Headache & 0 & 0 \\
\hline & Fibroid protruding through cervix & 0 & 0 \\
\hline & Infection of Lung & 0 & 0 \\
\hline & Thyroid cancer & 0 & 0 \\
\hline & Uterine bleed & 0 & $1(3 \%)$ \\
\hline & Within 4weeks after treatment & $1(3 \%)$ & $1(3 \%)$ \\
\hline & FroSPRMs, k 17- 38 & $1(3 \%)$ & $1(3 \%)$ \\
\hline \multirow{13}{*}{$\begin{array}{c}\text { Adverse } \\
\text { events }\end{array}$} & Changes. Hot flash & $7(23 \%)$ & $19(63 \%)$ \\
\hline & Headache & $5(16 \%)$ & $9(30 \%)$ \\
\hline & Procedural pain & $4(13 \%)$ & $3(10 \%)$ \\
\hline & Abdominal pain & $3(10 \%)$ & $4(13 \%)$ \\
\hline & Nausea & $2(6 \%)$ & $2(6 \%)$ \\
\hline & Fatigue & $2(6 \%)$ & $1(3 \%)$ \\
\hline & Anaemia & $1(3 \%)$ & $2(6 \%)$ \\
\hline & Nasopharyngitis & $1(3 \%)$ & $1(3 \%)$ \\
\hline & Acne & $2(6 \%)$ & $2(6 \%)$ \\
\hline & Breast pain or tenderness & $1(3 \%)$ & $1(3 \%)$ \\
\hline & Influenza & $1(3 \%)$ & $2(6 \%)$ \\
\hline & Insomnia & $1(3 \%)$ & $2(6 \%)$ \\
\hline & Pharyngitis & 0 & $1(3 \%)$ \\
\hline \multicolumn{4}{|c|}{ Table 3. Adverse Events (Safety Population) } \\
\hline
\end{tabular}

\section{Safety and Adverse Events}

\section{Primary End Points}

At $13^{\text {th }}$ week, median values of estradiol of the group receiving ulipristal acetate of $10 \mathrm{mg}$ were $60.5 \mathrm{pg}$ per millilitre (222 pmol per litre) but had reduced to postmenopausal levels in the leuprolide group (25.0 pg per millilitre [92 pmol per litre]) ( $\mathrm{P}<0.001$ for each ulipristal group vs. leuprolide acetate). The proportions of patients who reported moderate-to-severe hot flashes were $10 \%$ in the group receiving $10 \mathrm{mg}$ of ulipristal acetate, and $40 \%$ in the group receiving leuprolide acetate $(\mathrm{P}<0.001$ for both comparisons).

\section{Secondary End Points}

No significant differences were noted between the ulipristal group and the leuprolide groups regarding the proportion of patients who were reporting other adverse events or discontinuing treatment due to adverse events.

At the $13^{\text {th }}$ week, no clinically relevant differences were noticed among groups in corticotropin, thyrotropin, prolactin, or aminotransferase levels. There was a transient increase in 
the mean levels of total cholesterol (greater in the leuprolide group than in the ulipristal groups) during treatment. There were no significant group differences in blood pressure and heart rate at week 13.

At week 13, mean endometrial thicknesses were $10.7 \mathrm{~mm}$ in the group which were receiving $10 \mathrm{mg}$ of ulipristal acetate, and $5.1 \mathrm{~mm}$ in the group which were receiving leuprolide acetate $(\mathrm{P}<0.001$ for both comparisons).

No findings were observed in the endometrial biopsy examination. At week 13, benign endometrial changes were seen in all histologic specimens but for one patient in the group receiving $10 \mathrm{mg}$ of ulipristal acetate whose specimen showed simple hyperplasia. Adenocarcinoma or premalignant lesion findings were not found. Nonphysiologic endometrial changes were observed in 59 percent of those who were receiving $10 \mathrm{mg}$ of ulipristal acetate, and $12 \%$ of those who were receiving leuprolide acetate. At week 38, after 6 months of treatment-free follow-up among women who did not undergo hysterectomy or endometrial ablation, the frequency of nonphysiologic endometrial changes was low and similar in three study groups ( 6 to $7 \%$ ); all histologic specimens showed benign endometrium, except for one patient (in the leuprolide group) with simple hyperplasia.

\section{DISCUSSION}

Our study showed that daily use of $10 \mathrm{mg}$ oral ulipristal acetate was non-inferior to leuprolide acetate. Injection was given once in a month to reduce bleeding in patients who were planning treatment for fibroids. There was good response in all the study groups to treatment with PBAC scores of less than 75 at 13 weeks in $98 \%$ in patients receiving ulipristal acetate $10 \mathrm{mg} 89 \%$ in patients taking leuprolide acetate.

The uterine volume was observed to decrease in all three treatments and more predominantly in the leuprolide group than that of the ulipristal group. There was a considerable reduction of the largest fibroid volume in all three groups with no between-group significant differences.

Fibroids enlarged approximately after one month of the last dose of leuprolide acetate in a subgroup of patients who did not undergo surgery. Leiomyoma cell apoptosis might be the cause for the reduction of fibroid volume in majority of the patients who received ulipristal acetate for 6 months after the end of the treatment. ${ }^{11-13}$

Blood loss reduction with fibroids was also associated with an increase in haemoglobin and HCV in three study groups. ${ }^{14}$ These findings are consistent with improvement in anaemia and decreased uterine and fibroid volume of previous reports of leuprolide acetate. Ulipristal acetate decreased blood loss more rapidly than in patients taking leuprolide acetate due to its anti-proliferative action on the endometrium.

The mechanism of ulipristal acetate on fast effect on blood loss is uncertain and it may be due to direct effects on endometrium as it has anti-proliferative action. ${ }^{15,16}$

As with other SPRMs, ulipristal acetate induced benign changes in the endometrium. These findings had reversed when the patient was reassessed after 6 months without treatment, no dysplasia or neoplasia was identified among patients who were receiving ulipristal acetate. ${ }^{17-19}$ The treatments were similarly effective at reducing pain associated with fibroids and normalising quality of life.

In both ulipristal groups, levels of plasma estradiol were maintained in the mid-follicular range, whereas patients receiving leuprolide had on average a significant reduction to post-menopausal levels. Consistent with these findings, moderate to severe hot flashes were significantly less common with ulipristal acetate than with leuprolide acetate.

No clinically relevant effects of ulipristal acetate and leuprolide acetate on corticotropin, thyrotropin, prolactin, or glucose levels were noted. Four markers of bone turnover were elevated; median levels of one (CTX) were significantly lower at the end of treatment in both ulipristal groups than in the leuprolide group $\left(\mathrm{P}<0.001\right.$ for both comparisons. ${ }^{20,21}$ This finding indicates a higher rate of bone resorption in patients who were receiving leuprolide acetate than in those who were receiving ulipristal acetate, although we did not adjust for multiple testing.

In summary, in this randomized controlled study, we found that oral ulipristal acetate at a dose of $10 \mathrm{mg}$ was noninferior to monthly injections of leuprolide acetate in controlling uterine bleeding in women with symptomatic fibroids before planned surgery and had a better side effect profile.

\section{CONCLUSIONS}

Daily therapy of $10 \mathrm{mg}$ ulipristal acetate was non inferior to monthly injections of leuprolide acetate in controlling uterine bleeding and ulipristal acetate therapy was also significantly less likely to cause hot flashes.

\section{Limitations}

Several limitations were present in the study. It was not specifically designed to assess surgical outcomes, but rates and types of surgery were similar in the three study groups. As per clinical practice, uterine and fibroid volumes were not confirmed by central reading. In addition, the duration of treatment was restricted to 13 weeks. Hence, more data are needed regarding the benefits and risks of long-term treatment with ulipristal acetate.

Data sharing statement provided by the authors is available with the full text of this article at jemds.com.

Financial or other competing interests: None.

Disclosure forms provided by the authors are available with the full text of this article at jemds.com.

\section{REFERENCES}

[1] Donnez J, Donnez O, Dolmans MM. Safety of treatment of uterine fibroids with the selective progesterone receptor modulator, ulipristal acetate. Expert Opin Drug Saf 2016;15(12):1679-86.

[2] Donnez J, Dolmans MM. Uterine fibroid management: from the present to the future. Hum Reprod Update 2016;22(6):665-86. 
[3] Donnez J, Arriagada P, Donnez O, et al. Current management of myomas: the place of medical therapy with the advent of selective progesterone receptor modulators. Curr Opin Obstet Gynecol 2015;27(6):42231.

[4] Donnez J, Hudecek R, Donnez O, et al. Efficacy and safety of repeated use of ulipristal acetate in uterine fibroids. Fertil Sertil 2015;103(2):519-27.e3.

[5] Sayed GH, Zakhera MS, El-Nashar SA, et al. A randomized clinical trial of a levonorgestrel-releasing intrauterine sys- tem and a low-dose combined oral contra- ceptive for fibroid-related menorrhagia. Int J Gynaecol Obstet 2011;112(2):126-30.

[6] Viswanathan M, Hartmann $K$, McKoy $N$, et al. Management of uterine fibroids: an update of the evidence. Evid Rep Technol Assess (Full Rep) 2007;(154):1-122.

[7] Safrai M, Chill HH, Salzman AR, et al. Selective progesterone receptor modulators for the treatment of uterine leiomyomas. Obstet Gynecol 2017;130(2):315-8.

[8] Murji A, Whitaker L, Chow TL, et al. Selective progesterone receptor modulators (SPRMs) for uterine fibroids. Cochrane Db Syst Rev 2017;4(4):CD010770.

[9] Kalampokas T, Kamath M, Boutas I, et al. Ulipristal acetate for uterine fibroids: a systematic review and meta-analysis. Gynecol Endocrinol 2016;32(2):91-6.

[10] Pérez-López FR. Ulipristal acetate in the management of symptomatic uterine fibroids: facts and pending issues. Climacteric 2015;18(2):177-81.

[11] Yin P, Lin Z, Reierstad S, et al. Transcription factor KLF11 integrates progesterone receptor signaling and proliferation in uterine leiomyoma cells. Cancer Res 2010;70(4):1722-30.

[12] Yoshida S, Ohara N, Xu Q, et al. Cell-type specific actions of progesterone receptor modulators in the regulation of uterine leiomyoma growth. Semin Reprod Med 2010;28(3):260-73.
[13] Luo X, Yin P, Coon JS, et al. The selective progesterone receptor modulator CDB4124 inhibits proliferation and induces apoptosis in uterine leiomyoma cells. Fertil Steril 2010;93(8):2668-73.

[14] Sasaki H, Ohara N, Xu Q, et al. A novel selective progesterone receptor modulator asoprisnil activates tumor necrosis factor-related apoptosis-inducing ligand (TRAIL)- mediated signaling pathway in cultured human uterine leiomyoma cells in the absence of comparable effects on myometrial cells. J Clin Endocrinol Metab 2007;92(2):616-23.

[15] Kim JJ, Sefton EC. The role of progesterone signaling in the pathogenesis of uterine leiomyoma. Mol Cell Endocrinol 2012;358(2):223-31.

[16] Nieman LK, Blocker W, Nansel T, et al. Efficacy and tolerability of CDB-2914 treatment for symptomatic uterine fibroids: a randomized, double-blind, placeboconandtrolled, phase IIb study. Fertil Steril 2011;95(2):767-772.e2.

[17] Hoekstra AV, Sefton EC, Berry E, et al. Progestins activate the AKT pathway in leiomyoma cells and promote survival. J Clin Endocrinol Metab 2009;94(5):1768-74.

[18] Spitz IM. Clinical utility of progesterone receptor modulators and their effect on the endometrium. Curr Opin Obstet Gynecol 2009;21(4):318-24.

[19] Ioffe OB, Zaino RJ, Mutter GL. Endometrial changes from short-term therapy with CDB-4124, a selective progesterone receptor modulator. Mod Pathol 2009;22(3):450-9.

[20] Maruo T, Ohara N, Matsuo $\mathrm{H}$, et al. Effects of levonorgestrel-releasing IUS and progesterone receptor modulator PRM CDB-2914 on uterine leiomyomas. Contraception 2007;75(6 Suppl):S99-103.

[21] Xu Q, Ohara N, Liu J, et al. Selective progesterone receptor modulator asoprisnil induces endoplasmic reticulum stress in cultured human uterine leiomyoma cells. Am J Physiol Endocrinol Metab 2007;293(4):E1002-11. 